\title{
To the Content of Planck Units
}

\author{
Khachatur A. Kirakosyan \\ Institute of Chemical Physics, 5/2, Paruir Sevak St., Yerevan, Republic of Armenia, 0014 \\ Email: khkirakosyan@gmail.com
}

\begin{abstract}
Based on the slightly revised Planck system of units - the length, time, mass, temperature, and two dimensionless constants, one of which is the inverse of the fine structure constant, a new system of constants which is alternative to the system $c, h, G, k, m_{e}$ and $e$ is proposed: to the speed of light and constants of Planck, Newton, Boltzmann, the mass and charge of electron, respectively. It is shown that the Planck system of units has the same content with the coefficients of dimensionalities, which are introduced to give the corresponding dimensional content to physical quantities, and the constants $c, \hbar, G$ and $k$ are the combinations of the coefficients of dimensionalities.
\end{abstract}

Keywords: Electrons, constants, dimensions, Planck units.

\section{Introduction}

By generalized description of physical phenomena [1,2,3,4] along with the constants of Planck $h$, of Newton $G$, of Boltzmann $k$, the speed of light in vacuum $c$, the mass $m_{e}$ and the charge of the electron $e$, a key role also play the relations established between them:

$$
\begin{gathered}
\ell_{p}=\left(\frac{G \hbar}{c^{3}}\right)^{1 / 2}, t_{p}=\left(\frac{G \hbar}{c^{5}}\right)^{1 / 2}, \\
m_{p}=\left(\frac{c \hbar}{G}\right)^{1 / 2}, T_{p}=\left(\frac{\hbar c^{5}}{G k^{2}}\right)^{1 / 2} \\
\alpha=\frac{e^{2}}{c \hbar}
\end{gathered}
$$

where $\ell_{p}, t_{p}, m_{p}$ and $T_{p}$ is the Planck system of units of the length, time, mass and temperature, $\alpha$ is the fine structure constant, and $\hbar=h / 2 \pi$.

It is obvious that the creation of an alternative system of constants based on the unification of the permanent conceptual directions of physics and overlapping the essentially smaller intervals of space and time could become a tool for a more comprehensive and in-depth study of the physical world. However, the simple replacing the constants by the corresponding combinations of the Planck units does not bring to essential results. Apparently, due to this the constants $c, \hbar, G$ and $k$ and Planck units are included, according to NIST classification, into the category "the Universal constants" as the self-dependent constants [5]. Most likely, the impossibility to use the Planck units as primaries relative to $c, \hbar, G$ is caused by the uncertainty of their content.The unreasonable use of Planck units as characteristics values of the objects of investigations does not at all discloses the content of Planck units. The question is that Max Planck, with the aim of creation the universal system of units, unified the constants $c, \hbar, G, k$ based on their dimensionalities and, as was noted still by Bridgman [6], the results of relations (1) remain unclear by virtue of their origin, as well as remains unclear the content of the constant $\alpha$ itself $[7, ?]$.

In [9], by comparing the description of one and the same phenomena with the participation of the modelled microparticles and their conventional analogues, the conclusion has been made that the Planck units are the special coefficients introduced to give the dimensional content to the physical quantities.In this work we propose a new variant of the establishing the relations between constants $c, h, G$ and $k$, as well as between them and the mass and charge of electron. It is shown that the results of obtained relations can be used as a basis of a new system of constants which is alternative to the system $c, h, G, k, m_{e}$ and $e$. 


\section{Relations Between the Universal Constants of Physics and Mass of Electron}

Let a particle such as a photon, which is formed by the annihilation of slow electrons $e^{-}$and positrons $e^{+}$, be moving with the constant velocity and characterized by the constant path equal to the value of Compton length $\lambda_{c}$. Let the path traversed by the particle be measured by the number of strictly constant small intervals (of basis intervals), whose dimensional value is equal to $\xi_{d} \mathrm{~cm}$, respectively, the characteristic path of the particle is determined by

$$
H_{e 0}=\lambda_{c} / \xi_{d}
$$

the basis intervals.

Let the duration of the passage way $\xi_{d}$ be equal to $\xi_{\tau} c$, that is, we relate both path $\left(\xi_{d}\right)$, and time $\left(\xi_{\tau}\right)$ with the basis interval. The velocity of the given particle is the following

$$
c=\nu_{e 0} \lambda_{c}=\xi_{d} / \xi_{\tau},
$$

where $\nu_{e 0}$ is the frequency of the characteristic traversed path $\lambda_{c}$;

$$
\nu_{e 0}=\frac{1}{H_{e 0} \xi_{\tau}} .
$$

In view of (3) and (4) let us represent the right side of the identity $\lambda_{c} c \equiv \lambda_{c} c$ by the product of the dimensional and dimensionless components:

$$
\lambda_{c} c \equiv H_{e 0} \frac{\xi_{d}^{2}}{\xi_{\tau}} .
$$

Multiplying and dividing the right side of this equation by the constant $\xi_{m}$ with the dimension of the mass, we obtain

$$
\lambda_{c} c=h m_{e}^{-1} \quad \text { or } \quad m_{e} c \lambda_{c}=h .
$$

where it is designated:

$$
\begin{aligned}
& m_{e}=\frac{\xi_{m}}{H_{e}}, \\
& h=\frac{\xi_{m} \xi_{d}^{2}}{\xi_{\tau}},
\end{aligned}
$$

As soon as the Compton wavelength is associated with electron, taking the velocity (4) equal to the speed of light in vacuum, the equation (7) can be identified with the de Broglie equation as applied to the electron in the "rest", the mass of electron and the Planck constant are determined by the formulas (8) and (9), respectively. We emphasize that in this case $\lambda_{c}$ is not the wavelength but the real path of electron during the characteristic time $\tau_{0}=H_{e 0} \xi_{\tau}$.

Let the characteristic path length $\lambda_{c}$ and the circumference with the classic radius $r_{e}$ of electron be related by the equation

$$
\lambda_{c}=\alpha_{c} 2 \pi r_{e} \quad \text { or } \quad H_{e 0}=\alpha_{c} H_{e c}
$$

where $H_{e c}$ by analogy with the equation (3), is the number of basis intervals of circumference $2 \pi r_{e}=H_{e c} \xi_{d}$, $\alpha_{c}$ is the dimensionless constant.

Combining the equations (7) and (10) we obtain $m_{e} c \alpha_{c} r_{e}=\hbar$ and after multiplying both sides by the constant $c$ (4) with allowance of (2), we obtain

$$
m_{e} c^{2}=\frac{c \hbar}{\alpha_{c} r_{e}}=\frac{e^{2}}{r_{e}} .
$$

Comparing the pairs of equations (2), (4) and (9) we can conclude that $\alpha_{c}=\alpha^{-1}$, that is the proportionality factor $\alpha_{c}$ in the equation (10) is equal to the inverse of the fine structure constant. In [9], the quantity $\alpha_{c}$ is defined by the equation $\alpha_{c}=\alpha_{0} / \chi_{c}$, where $\alpha_{0}=\sum_{n=1}^{7} \sum_{l=0}^{n-1}(2 l+1)=140, n$ and $l$ 
are the analogues of the principal and azimuthal quantum numbers for given hierarchical level, $\chi_{c}$ is the dimensionless unit caused by the specificity of formation of the closed curvlinear trajectory.

From the definitions (3), (5) and (8) it follows that the multiplying of some dimensionless quantity by the constant with the symbol $\xi$, and assigning the appropriate dimension to it, the indices at $\xi$ indicate the common designation of dimensional parameter, that is, $\xi_{d}, \xi_{\tau}$ and $\xi_{m}$ are the coefficients of dimensions of length, time and mass, respectively. Hence, the constants with the symbol $\xi$ are called the coefficients (or operators) of dimensions.

Taking into account the formulas $(2),(4),(9)$ and denoting $\xi_{\tau}^{-1}=\xi_{\nu}$, one can combine the coefficient of dimension of energy $\xi_{\varepsilon}$ by various variants

$$
\xi_{\varepsilon}=\frac{\xi_{m} \xi_{d}^{2}}{\xi_{\tau}^{2}}=\frac{c h}{\xi_{d}}=h \xi_{\nu}=\frac{2 \pi \alpha_{c} e^{2}}{\xi_{d}}
$$

In turns, dividing the listed variants of definition $\xi_{\varepsilon}$ by $H_{e 0}$, the energy of electron will be represented in the following form

$$
\frac{\xi_{\varepsilon}}{H_{e 0}}=m_{e} c^{2}=\frac{c h}{\lambda_{c}}=h \nu_{e 0}=\frac{e^{2}}{r_{e}}
$$

that is, depending on the mass (8), characteristic path (3), the frequency of revealing of characteristic path $\lambda_{c}(5)$ and as a result of electrostatic interaction at the distance $r_{e}$ (13). From the equations (8) and (13) the unity of mass and energy of electron follows directly: both the mass, and the energy of the electron are the dimensional manifestations of the one and the same the dimensionless constant $H_{e 0}$. It should also be noted that writing the energy as $\varepsilon=h \nu_{e 0}$, the parameter $\nu_{e 0}$ should be not considered as a frequency of the wave conjugate with the particle, but as a frequency of the manifestation of its characteristic path $\lambda$.

Given the formulas $(2),(4),(8)$ and $(9)$, the ratio of electrostatic $\left(F_{q}\right)$ and gravitational $\left(F_{G}\right)$ interactions of two electrons at the equal distance will be represented as

$$
\left(\frac{F_{q}}{F_{G}}\right)_{r}=\frac{e^{2}}{G m_{e}^{2}}=\frac{\xi_{m} \xi_{d}^{3} H_{e 0}^{2}}{G 2 \pi \alpha_{c} \xi_{\tau}^{2} \xi_{m}^{2}}=\frac{H_{e 0}^{2}}{\alpha_{c}}=\alpha_{c} H_{e c}^{2}
$$

where by virtue of the fact that the ratio of two parameters of the same content and the same dimension must be the dimensionless quantity, is denoted

$$
\frac{\xi_{m} \xi_{d}^{3}}{G 2 \pi \xi_{\tau}^{2} \xi_{m}^{2}}=1 \quad \text { or } \quad G=\frac{\xi_{d}^{3}}{2 \pi \xi_{m} \xi_{\tau}^{2}}
$$

Note that Eddington had called the ratio $\left(F_{q} / F_{G}\right)_{r}$ as the constant of the Universe.

If to write the Boltzmann constant $k$ as a combination of $\xi_{\varepsilon}(12)$ and the coefficient of temperature $\xi_{T}$, that is, $k=\xi_{\varepsilon} / \xi_{T}$ and using the equations (4), (8), (9) and (15) the numerical values of the dimensional coefficients will be defined by the formulas

$$
\begin{gathered}
\xi_{d}=\left(\frac{2 \pi G h}{c^{3}}\right)^{1 / 2}, \quad \xi_{\tau}=\left(\frac{2 \pi G h}{c^{5}}\right)^{1 / 2}, \\
\xi_{m}=\left(\frac{c \hbar}{G}\right)^{1 / 2}, \quad \xi_{T}=\left(\frac{\hbar c^{5}}{G k^{2}}\right)^{1 / 2}, \\
\xi_{d}=\frac{h}{H_{e o} m_{e} c}, \quad \xi_{\tau}=\frac{h}{H_{e o} m_{e} c^{2}} \\
\xi_{m}=m_{e} H_{e o}, \quad \xi_{T}=\frac{H_{e o} m_{e} c^{2}}{k}
\end{gathered}
$$




\section{The Dimentional Coefficients and Planck's Units}

From the comparison of the equations (1) and (16) it follows that after the slight revision of $\ell_{p}$ and $\ell_{t}$, the formula for definition the Planck system of units and the dimensional coefficients coincide: $\xi_{d}=\ell_{p}^{*}=2 \pi \ell_{p}, \xi_{\tau}=t_{p}^{*}=2 \pi t_{p}, \xi_{m}=m_{p}, \xi_{T}=T_{p}$, thus the role of dimensional coefficients can be ascribed to the Planck system of units.

Using the formulas $(2),(8),(9)$ and the series (17), some known quantities using the constants $H_{e c}, \alpha_{c}$ and the corresponding dimensional coefficients will be represented as

- the radii of Bohr of the hydrogen atom

$R_{n}=\frac{2 n^{2} \hbar^{2}}{m e^{2}}=\frac{n^{2} \alpha_{c}^{2} H_{e c} \xi_{d}}{2 \pi}$,

- the energy of an electron at the Bohr orbit

$\varepsilon_{n}=\frac{m e^{4}}{2 n^{2} \hbar^{2}}=\frac{\xi_{\varepsilon}}{2 H_{e 0} \alpha_{c}^{2} n^{2}}$

- the classical electron radius

$r_{e}=\frac{e^{2}}{m_{e 0} c^{2}}=\frac{H_{e c} \xi_{d}}{2 \pi}$

- the second constant of radiation

$c_{2}=c h / k=\xi_{d} \xi_{T}=\ell_{p}^{*} T_{p}$,

- the Boltzmann definition of entropy

$S=k \ln W=\frac{\xi_{\varepsilon}}{\xi_{T}} \ln W$,

where $W$ is the thermodynamic probability.

From the equations (3), (5), (8) and the adduced examples it follows that many physical quantities are the products of dimensional coefficients and dimensionless components that most likely are the carriers of information on the physical contents of the given quantity (of the structural or other character). Thus, in view of the equality of $m_{p}$ and $\xi_{m}$ it follows that the gravitational coupling constant $\chi_{G}$ [5] is determined by the relation $\left(m_{e} / \xi_{m}\right)^{2}$ which is equal to the square of the inverse value of $H_{e o}^{2}$. From this point of view one should expect that the dimensionless constants obtained in [10] by the establishment of relations between the Planck mass and the constants of energy of the Standard Model, would acquire a new and highly promising content.

\section{Conclusions}

Based on the above, one can conclude that the systems of constants $c, \hbar, G, k, e, m_{e}$ and $\ell_{p}^{*}, t_{p}^{*}, m_{p}$, $T_{p}, \alpha_{c}, H_{e c}$ are fully interchangeable, the Planck system of units are the coefficients of dimensionalities, which are introduced to give the dimensional content to dimensionless quantities and the constants $c, \hbar$, $G$ and $k$ are the combinations of the coefficients of dimensionalities.

There are sizes in Nature but not the dimensionalities; accordingly, the most adequate models of the surrounding world should be dimensionless, too. Just these are the Planck units with the content attributed to them becoming the key to the transition to the dimensionless description of physical quantities.

\section{References}

1. S. WeinBerg, The Quantum Theory of Fields. (Cambridge Univ. Press, USA, 1996), p. 514.

2. M. D. Schwartz, Quantum Field Theory and the Standard Model. (Cambridge Univ. Press, USA, 2013), p. 851.

3. M. B. Green, J. H. Schwartz, and E. Witten, Superstring Theory. (Cambridge Univ. Press, Cambridge,1987), p. 482.

4. K. Becker, M. Becker, and J. Schwartz, String Theory and M - Theory. A Modern Introduction. Cambridge Univ.Press, ISBN-0-52186069-5, 2007, p. 739.

5. The NIST Reference on Constants, Units, and Uncertainty. CODATA Internationally recommended, 2014 values of the Fundamental Physical Constants. http://physics.nist.gov/cuu/Constants/ (2016).

6. P. W. Bridgman, Dimensional analysis. (Yale University Press, New Haven and London, 1963),p. 113. 
7. R. P. Feynman, QED, The Strange Theory of Light and Matter. Princeton, N-JPrinceton Univ. Press, 1985, p. 163.

8. H. Kragh, Archive for History of Exact Sciences. 57, p. 395-431, (2003).

9. Kh. A. Kirakosyan, Structural Physics. Structural Content of the Basic Concepts and Physical Laws. Moscow, Flinta, ISBN 978-5-9765-1087-6, 2011, p. 420 (in Russian.)

10. F. Wilczek, Physics Today. 59, 10-11 [MIT-CTP-3742], (2006). 